\title{
Cohesive-Zone Effects in Hydraulic Fracture Propagation
}

\author{
Dmitry I. Garagash \\ August 21, 2019 \\ Dept. Civil and Resource Engng., Dalhousie Univ., Halifax, Canada \\ garagash@dal.ca
}

\begin{abstract}
Hydraulic fracture presents an interesting case of crack elasticity and fracture propagation non-linearly coupled to fluid flow. Hydraulic fracture (HF) is often modeled using the Linear Elastic Fracture Mechanics (LEFM), which assumes that the damaged zone associated with the rock breakage near the advancing fracture front is small compared to the lengthscales of other physical processes acting during propagation. The latter include dissipation in viscous fluid flow in the fracture channel, of which the fluid lag - a region adjacent to the fracture tip filled with fracturing fluid volatiles and/or infiltrated formation pore fluid - is the extreme manifestation. In this study we address the validity of the LEFM approach to hydraulic fracturing by constructing the solution for the near tip region of a cohesive fracture driven by Newtonian fluid in an impermeable linear-elastic rock. We show that the near HF tip solution has an intricate structure supported by a number of nested lengthscales on which different dissipation processes are realized. This structure is bookended by the solid ' $c$ ' or fluid lag ' $o^{\prime}$ process zone immediately near the tip and by the viscosity ' $m$ ' asymptote away from the tip, while the LEFM ' $k$ ' asymptote may emerge at intermediate distances within the $c / o$ to $m$ transition. Realization of the $k$ asymptote, and, therefore, the viability of the LEFM in HF, depends on two parameters: the cohesive-to-fluid-lag fracture energy ratio $G_{c} / G_{o}$ and the cohesive-to-in-situ stress ratio $\sigma_{c} / \sigma_{o}$. For representative values of the former, we show that the LEFM-behavior emerges only when the cohesive stress is large compared to the in situ confining. Since $\sigma_{c} \sim$ few MPa for most rocks, it follows that the LEFM may only be applicable to laboratory hydraulic fracture conducted under low confining stress, and, conversely, is not applicable to the field hydraulic fracturing characterized by larger values of $\sigma_{o}$. We further use an approximate 'equation of motion' approach based on the continuation of the tip solution onto the entire fracture to solve for the propagation of a penny-shape HF driven by a point source fluid injection and quantify the prominence of the non-LEFM effects.
\end{abstract}

Keywords: fracture mechanics; hydraulic fracture; cohesive zone; fluid lag; Gauss-Chebyshev quadrature; equation of motion

\section{Introduction}

Problem of steady propagation of a semi-infinite hydraulic fracture allows for (i) assessing the coupling of various physical processes manifested near the fracture tip and their effect on the HF propagation, and (ii) development of hydraulic fracture propagation logic/criteria that can be integrated into the numerical framework for solution of finite hydraulic fracture propagation (e.g. $[1,2,3])$. The aforementioned physical processes of primary interest include the solid and fluid process zones adjacent to the fracture front, which both contribute to the energy dissipation during the fracture advance. The former corresponds to the damaged region where the rock strength/cohesion degrades with the increasing fracture surfaces separation $[4,5,6,7]$, and the latter corresponds to the so-called fluid lag, the region near the fracture tip saturated by the fracturing fluid volatiles and/or infiltrated pore fluid from the surrounding rock characterized by maximum fluid pressure drop in the fracture (e.g., $[8,9])$. The viscous fluid flow in the fracture channel outside of the near tip solid/fluid process zones also dissipates the mechanical energy, and, in fact, is expected to dominate when the fracture length is much larger than the process zone(s) size (e.g., $[9,10,11])$. 
Many HF modeling efforts to date have utilized the premise of the Linear Elastic Fracture Mechanics (LEFM) (see review [12] and references therein), which assumes that the solid process zone can be lumped into a crack tip singularity characterized by a finite energy release rate (cohesive fracture energy). The latter, so-called small scale yielding assumption [13] requires the cohesive zone to be small compared to the lengthscales of other physical processes active during the HF propagation, including that of the fluid lag. Rubin [8] appears to be the first to study the interplay of the solid and fluid process zones in a fluid-driven fracture in application to magmatic dike propagation. Very high magma fluid viscosity leads to extensive fluid pressure drop region in the flow towards the propagating fracture tip, resulting in the fluid lag size much larger than the cohesive zone size if the typical laboratory values of the cohesive fracture energy are assumed. In this case, the small scale yielding assumption would then seem to apply, validating the applicability of the LEFM. However, in industrial hydraulic fracturing applications, the fracturing fluid viscosity may not be high enough to separate the fluid and solid process zones scales, thus necessitating the full analysis of the near tip region of a cohesive hydraulic fracture.

Several authors have numerically examined the effects of the cohesive zone on hydraulic fracture propagation using a finite element approach $[14,15,16,17]$. All these studies have assumed a zero fluid lag, which as we show here should result in a non-integrable fluid pressure singularity at the fracture tip, making the mathematical problem ill-posed, if the classical lubrication approximation (Pouiseulle law) is used to describe the fluid flow. Sarris and Papanastasiou $[14,15]$ have circumvented this problem by amending the Pouiseulle law by an ad-hoc constant permeability law in the cohesive part of the fracture, and provided numerical examples of cohesive zone effects on the hydraulic fracture propagation.

In this work we propose a systematic parametric study of the HF tip propagation problem (Figure 1) accounting for the coupling of the solid and fluid process zones for the case of a fracture driven by a Newtonian fluid in an impermeable rock, while assuming the classical lubrication approximation for the fluid flow. We first use asymptotic analysis to suggest the structure of the solution, as it is supported by a number of nested lengthscales, on which different dissipation processes and corresponding limiting solutions are realized. We then solve the problem numerically using an extension of the numerical method recently proposed in [18]. This solution, when fully characterized in the problem parametric space, enables to assess the the significance of the non-LEFM coupling between between the fluid and solid process zones and whether/when the LEFM behavior may emerge in hydraulic fracture.

In order to demonstrate how the near tip cohesive HF effects (encapsulated in the solution of a semi-infinite HF model) translate to the propagation of a finite hydraulic fracture, we will consider the propagation of an axisymmetric (penny-shape) HF from a point fluid source. Full numerical solution for the propagation of a finite hydraulic fracture can be challenging even for fractures of simple (one-dimensional) geometry. A robust approach to solving this problem, which allows bridging between the physical lengthscales of the tip processes and the finite fracture scale using the embedment of the tip solution (such as the one developed here) into the tip logic of the numerical method for the finite fracture (e.g., $[1,2,3])$, has been so far implemented for the LEFM-based propagation with no fluid lag. Here we will use a simpler approach to approximate solution for propagation of finite HF based on a suitable continuation of the tip solution onto the entire fracture. This approach, which leads to an ordinary differential 'equation-of-motion' for the fracture trajectory (fracture length as a function of time), has been recently used successfully by Dontsov $[19,20]$ to construct approximate solutions for finite fractures under more the restrictive, LEFM and no-fluid-lag assumptions.

The paper is organized as follows. First, we present the mathematical modeling framework for steady propagation of a semi-infinite fluid-driven cohesive fracture that is used to describe the near tip region of an arbitrary finite HF. We then discuss various asymptotic reductions of this framework, including the LEFM (or small scale yielding) limit, and the corresponding limiting near tip solutions, which allow to frame the general solution for the cohesive HF tip developed next. Finally, we use the continuation of the near tip solution onto the entire finite fracture to introduce the latter equation of motion and solve it for the case of the HF of radial geometry. We finish with a discussion of the non-LEFM effects in the HF propagation and conclusions.

\section{Cohesive hydraulic fracture tip problem formulation}

Integral equation of linear elasticity relates the crack opening $w$ to the normal stress change along the crack - from its ambient value $\sigma_{o}$ to the current value set by the difference between the fluid/volatile pressure in 
the crack $p_{f}$ and the cohesive stress $\sigma_{c o h}$ :

$$
p_{f}(x)-\sigma_{c o h}(w(x))-\sigma_{o}=\frac{E^{\prime}}{4 \pi} \int_{0}^{\infty} \frac{d w}{d s} \frac{d s}{x-s} \quad(x>0)
$$

where $x$ is the distance from the steadily advancing crack tip (Figure 1b), and $E^{\prime}=E /\left(1-\nu^{2}\right.$ ) is the plane strain modulus. The cohesive stress weakens with the increasing crack opening from peak value $\sigma_{c}$ at $w=0$

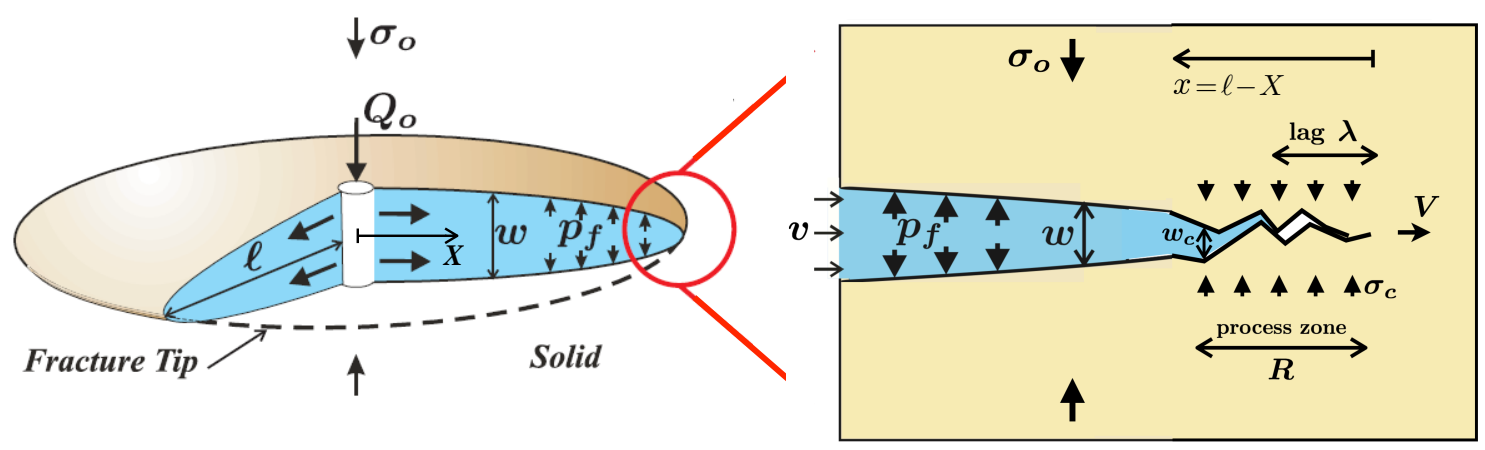

Figure 1: Sketch of a finite (e.g. radial) hydraulic fracture, (a), and its near-tip region modeled as a semiinfinite crack, (b).

to zero at/above some critical cohesive crack opening $w \geq w_{c}$. The length of the process zone $R$ is then given by the distance from the tip where the critical opening is reached, i.e. $w(R)=w_{c}$. Here, we will make use of the piecewise linear relation, $\sigma_{c o h}(w)=\sigma_{c}\left(1-w / w_{c}\right)$ when $w<w_{c}$ and zero otherwise [6, 7]. The corresponding cohesive fracture energy is

$$
G_{c}=\int_{0}^{\infty} \sigma_{c o h}(w) d w=\frac{1}{2} \sigma_{c} w_{c}
$$

When considering a viscous fluid and its flow in the fracture, the inability of the fluid to catch up with the moving crack front results in the fracture tip cavity ("lag") of extent $\lambda$ filled by the fracturing fluid volatiles or by the pore fluid and its volatiles sucked-in from the formation. The fluid pressure in the tip cavity

$$
p_{f}(x)=p_{o} \quad(0<x<\lambda)
$$

can therefore range from near zero (saturated vapor pressure), when compared to the representative stress value $\sigma_{o}$, up to the virgin formation pore pressure value. Assuming the former, the fluid pressure drop $\sigma_{o}-p_{o} \approx \sigma_{o}$ in the fluid lag suggest a measure of the associated energy dissipation, which we term the "lag fracture energy"

$$
G_{o}=\sigma_{o} w_{o}
$$

where $w_{o} \sim\left(\sigma_{o} / E^{\prime}\right) \lambda$ is the characteristic fracture opening in the lag, that can be more formally defined by replacing $\lambda$ with the lengthscale $\ell_{0}$ defined in the next section.

Conservation of the incompressible fracturing fluid in the rest of the fracture, $v=V$, where $V$ is the crack tip velocity, together with the Poiseuille law for a Newtonian fluid leads to (e.g. [10])

$$
V=\frac{w^{2}}{\mu^{\prime}} \frac{d p_{f}}{d x} \quad(x>\lambda)
$$

with a fluid viscosity parameter $\mu^{\prime}=12 \mu$. The above equations govern the solution for the HF tip problem in terms of the distribution of the fracture opening $w(x)$, net fluid pressure $p(x)=p_{f}(x)-\sigma_{o}$, and the extent of the lag $\lambda$.

We note in passing that the presence of the non-zero fluid lag is not only physical, but, in fact, a mathematical necessity in the cohesive HF. Indeed, fracture cohesion requires finite (non-singular) stress in 
the elastic solid embedding the crack, and, by extension, finite fluid pressure (as a part of the traction acting on the fracture walls) in the fracture. Under these conditions, the crack elasticity requires that the fracture opens smoothly, $w \propto x^{3 / 2}$, which, in view of the Poiseuille law (when extended all the way to the fracture tip under zero lag assumption) leads to non-integrable pressure singularity, $d p_{f} / d x \propto 1 / x^{3}$ as $x \rightarrow 0$. The inclusion of the Carter's leak-off of the fracturing fluid into a permeable rock, modifies the lubrication flow equation (2) by addition leak-off term $C^{\prime} \sqrt{V x} / w$ in the left hand side [21], thus, leading to even stronger pressure singularity $d p_{f} / d x \propto 1 / x^{4}$ as $x \rightarrow 0$ when the the lag is assumed to be zero.

\section{$2.1 \quad$ LEFM limit}

The above cohesive hydraulic fracture formulation reduces to the Linear Elastic Fracture Mechanics (LEFM) one in the limit when the cohesive zone is lumped into a point singularity (at the fracture tip) characterized by $\sigma_{c} \rightarrow \infty, w_{c} \rightarrow 0$, and finite cohesive fracture energy $G_{c}=\sigma_{c} w_{c} / 2<\infty$. Or, alternatively, the cohesive stress in the LEFM limit is given by a Dirac-delta function of the crack opening,

$$
\sigma_{c o h}(w)=G_{c} \delta_{\operatorname{Dirac}}(w)
$$

In this case, the cohesive stress is eliminated from the crack elasticity equation (1), while the fracture propagation condition can be written in the form of the classical LEFM crack tip asymptote [13]

$$
x \rightarrow 0: \quad w=\frac{K_{I c}}{E^{\prime}} \sqrt{\frac{32 x}{\pi}}
$$

where the critical stress intensity factor (fracture toughness) $K_{I c}$ is related to the cohesive fracture energy $K_{I c}=\sqrt{G_{c} E^{\prime}}$.

\section{Asymptotic behavior and existing crack tip solutions}

A number of different asymptotic behaviors that can be realized near the tip of a hydraulic fracture are listed in the following for the fracture opening $w$ and the net fluid pressure $p=p_{f}-\sigma_{o}$ :

$$
\begin{gathered}
m \text {-asymptote : } \quad w=\beta_{m} \ell_{m}^{1 / 3} x^{2 / 3} \quad \frac{p}{E^{\prime}}=\beta_{m} \frac{\cot (-\pi / 3)}{4} \frac{\ell_{m}^{1 / 3}}{x^{1 / 3}} \\
k \text {-asymptote: } w=\beta_{k} \ell_{k}^{1 / 2} x^{1 / 2} \quad \frac{p}{E^{\prime}}=\frac{1}{\beta_{k}^{2}} \frac{\ell_{m}}{\ell_{k}} \ln x+\text { const } \\
o \text {-asymptote: } \quad w=\beta_{o} \frac{\sigma_{o}}{E^{\prime}} \frac{x^{3 / 2}}{\ell_{o}^{1 / 2}} \quad p=-\sigma_{o} \\
c \text {-asymptote: } w=\beta_{c} \frac{\sigma_{c}}{E^{\prime}} \frac{x^{3 / 2}}{\ell_{c}^{1 / 2}} \quad p=0
\end{gathered}
$$

where prefactors are: $\beta_{k}=4 \sqrt{2 / \pi}$ [13], $\beta_{m}=2^{1 / 3} 3^{5 / 6}[10], \beta_{o} \approx 1.796[9,18]$, and $\beta_{c} \approx 4.3016[18]$ and lengthscales are defined by

$$
\ell_{m}=\frac{\mu^{\prime} V}{E^{\prime}} \quad \ell_{k}=\frac{G_{c}}{E^{\prime}} \quad \ell_{c}=\frac{E^{\prime}}{\sigma_{c}} w_{c} \quad \ell_{o}=\left(\frac{E^{\prime}}{\sigma_{o}}\right)^{3} \ell_{m}
$$

The $m$ asymptote corresponds to the fracture propagation dominated by the dissipation in the lubrication fluid flow. Since the latter is the only dissipation process that is not localized to the fracture tip (i.e. dissipation in the viscous fluid flow persists with the distance from the tip, which can not be said about the dissipation in the solid and fluid process zones), the $m$ asymptote is always realized at large enough distances from the tip. Thus, the near tip hydraulic fracture solution can be framed as a sequence of various asymptotes listed in the above realized over their corresponding lengthscales (as long as those separate), transitions between them, and eventual transition to the far field $m$ asymptote. 
We define the candidate transitional lengthscales in the spirit of Garagash et al. [21], such that at the distances from the tip $\sim$ the transitional lengthscale the corresponding pair of asymptotic expressions for the opening yield similar (order of magnitude) values. Specifically, we get

$$
\ell_{k m}=\frac{\ell_{k}^{3}}{\ell_{m}^{2}} \quad \ell_{m c}=\left(\frac{E^{\prime}}{\sigma_{c}}\right)^{6 / 5} \ell_{m}^{2 / 5} \ell_{c}^{3 / 5} \quad \ell_{k o}=\frac{E^{\prime}}{\sigma_{o}} \ell_{k}^{1 / 2} \ell_{o}^{1 / 2} \quad \ell_{c k}=\ell_{c} \quad \ell_{o m}=\ell_{o}
$$

for the toughness-viscosity $(k \rightarrow m)$, viscosity-cohesion $(m \rightarrow c)$, and toughness-lag $(k \rightarrow o)$ candidate transitions, respectively. We also added to the list (9) the two previously defined lengthscales, $\ell_{c}$ and $\ell_{o}$, which correspond to the process zone transition scales for the solid $(c \rightarrow k)$ and for the fluid $(o \rightarrow m)$, respectively.

A particular scaling of the HF tip solution can be devised by using one of the above transitional scales for the distance from the tip, the corresponding stress/pressure scale, and the corresponding elastic scale for the crack opening. For example, in the $\ell_{o}$-scaling, the lag lengthscale $\ell_{o}$, the pressure scale $\sigma_{o}$, and the opening scale $w_{o}=\left(\sigma_{o} / E^{\prime}\right) \ell_{o}$ are used to normalized the distance from the tip $x$, net pressure $p$, and crack opening $w$, respectively (Appendix A).

The normalized solution in any such scaling can be shown to be a function of two non-dimensional parameters

$$
\Sigma_{c}=\frac{\sigma_{c}}{\sigma_{o}} \quad \Gamma_{c}=\frac{G_{c}}{G_{o}}=\frac{1}{2} \frac{w_{c}}{w_{o}} \frac{\sigma_{c}}{\sigma_{o}}
$$

the cohesive-to-confining stress ratio and the cohesive-to-lag fracture energy ratio, respectively. The transitional scales normalized by $\ell_{0}$ can be expressed in terms of the above two non-dimensional parameters as

$$
\frac{\ell_{o}}{\ell_{o}}=1 \quad \frac{\ell_{k o}}{\ell_{o}}=\Gamma_{c}^{1 / 2} \quad \frac{\ell_{m c}}{\ell_{o}}=\Sigma_{c}^{-12 / 5}\left(2 \Gamma_{c}\right)^{3 / 5} \quad \frac{\ell_{c}}{\ell_{o}}=\Sigma_{c}^{-2} 2 \Gamma_{c} \quad \frac{\ell_{k m}}{\ell_{o}}=\Gamma_{c}^{3}
$$

In the following we review some of the known limiting crack tip solutions before proceeding with the general solution of the problem.

\subsection{Dry crack tip with a cohesive process zone}

This is a classical fracture mechanics solution [13], which corresponds to the $c \underset{\ell_{c}}{k} k$ transition from the cohesive-stress-dominated near field asymptote (7) to the far (or intermediate) field LEFM asymptote (5) over distances from the tip $\sim \ell_{c}$. In the context of the hydraulic fracture, this solution can be realized when the cohesive zone is deeply embedded within fluid lag, i.e. $\ell_{c} \ll \ell_{o}$, which is expected when cohesive fracture energy is small $G_{c} / G_{o} \ll 1$ and the peak cohesive stress is large $\sigma_{c} / \sigma_{o} \gg 1$.

\subsection{The LEFM HF tip with the fluid lag}

Garagash and Detournay [9] provide the HF tip solution under provisions of the LEFM, i.e. when the cohesive zone is lumped into a point singularity characterized by $\sigma_{c} / \sigma_{o} \rightarrow \infty, w_{c} / w_{o} \rightarrow 0$, and fixed (finite) cohesive fracture energy $G_{c}=\sigma_{c} w_{c} / 2<\infty$. Thus, the solution depends on the single non-dimensional parameter, the normalized fracture energy $\Gamma_{c}=G_{c} / G_{o}$. The latter is related to the non-dimensional fracture toughness parameter $\kappa$ used in [9] by $\kappa=\sqrt{32 \Gamma_{c} / \pi}$. The solution, shown in the $\ell_{o}$-scaling on Figure 2a, is characterized by the near tip $k$ and the far-field $m$ behavior. The two limiting solution regimes correspond to

$$
G_{c} / G_{o} \gtrsim 1: \quad k \underset{\ell_{k m}}{\rightarrow} m, \quad G_{c} / G_{o} \ll 1: \quad k \underset{\ell_{k o}}{\rightarrow} \stackrel{\rightarrow}{\ell_{o}} m
$$

for the case of vanishing lag $\left(\Gamma_{c} \gtrsim 1\right)$ when the direct transition from the $k$ to $m$ asymptote happens over distances $\sim \ell_{k m}$ and the case of vanishing toughness $\left(\Gamma_{c} \ll 1\right)$ when the lag $o$-asymptote develops at intermediate distances from the tip, respectively. Figure 4a provides a complimentary presentation of the fracture opening profiles (Figure 2a), when normalized by the far-field $m$-asymptote. Evolution of the fluid lag between its maximum value at zero toughness to exponentially vanishing values [9] at large toughness is shown on Figure 3 (marked by 'LEFM'). 

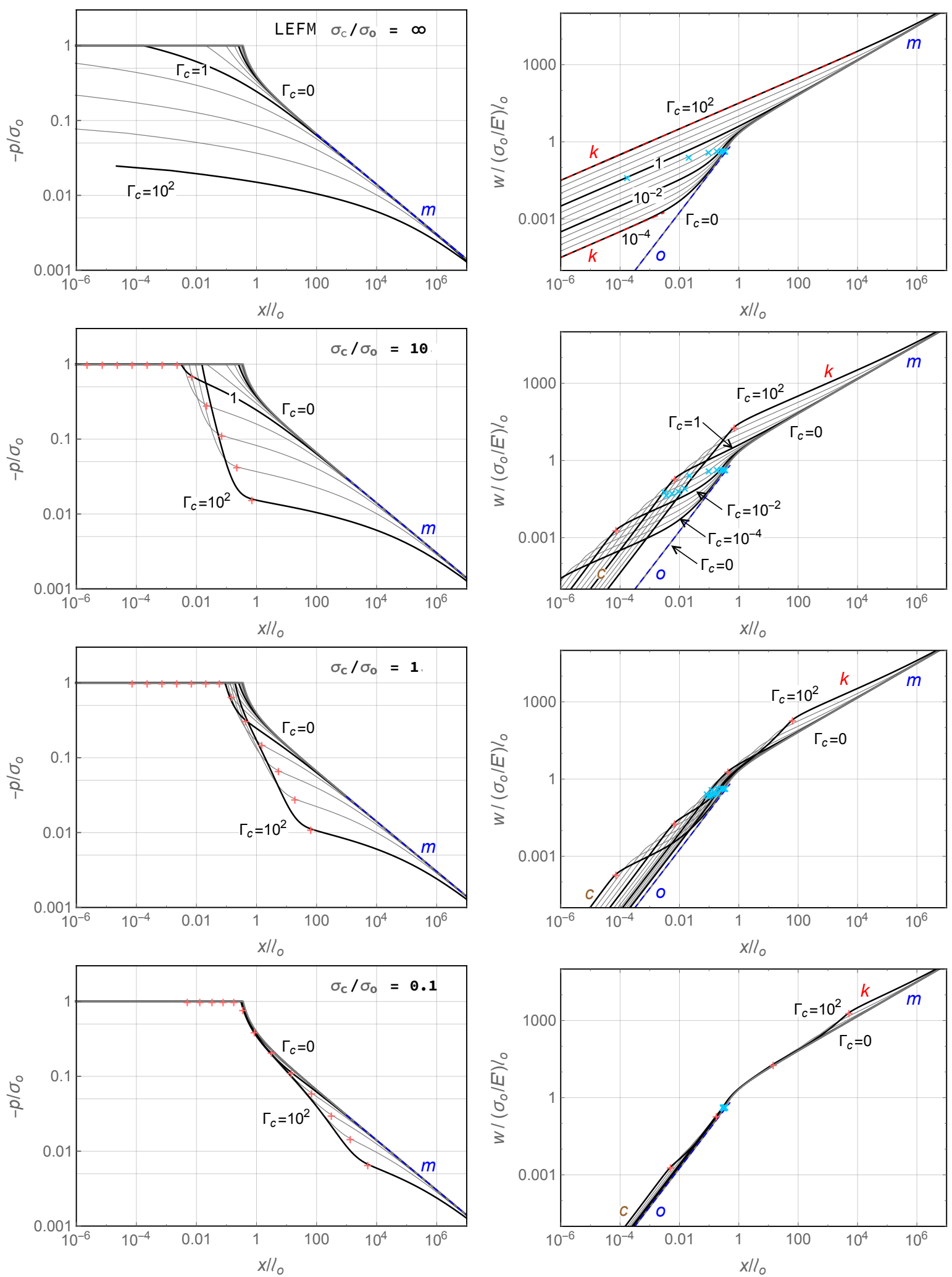

Figure 2: HF tip solutions for the net pressure (left) and crack opening (right) for $\sigma_{c} / \sigma_{o}=$ $\infty$ (LEFM), 10, 1, 0.1 and various values of the normalized cohesive fracture energy $\Gamma_{c}=G_{c} / G_{o}$ between $10^{-4}$ to $10^{2}$ in $10^{0.5}$ increments on the logarithmic scale. The zero toughness $\left(\Gamma_{c}=0\right)$ solution is also shown. The extent of the fluid lag $\lambda$ and of the cohesive zone $R$ in the fracture are shown by $\times$ and + markers. The various asymptotic behaviors $(c, o, k, m)$ are also indicated. 

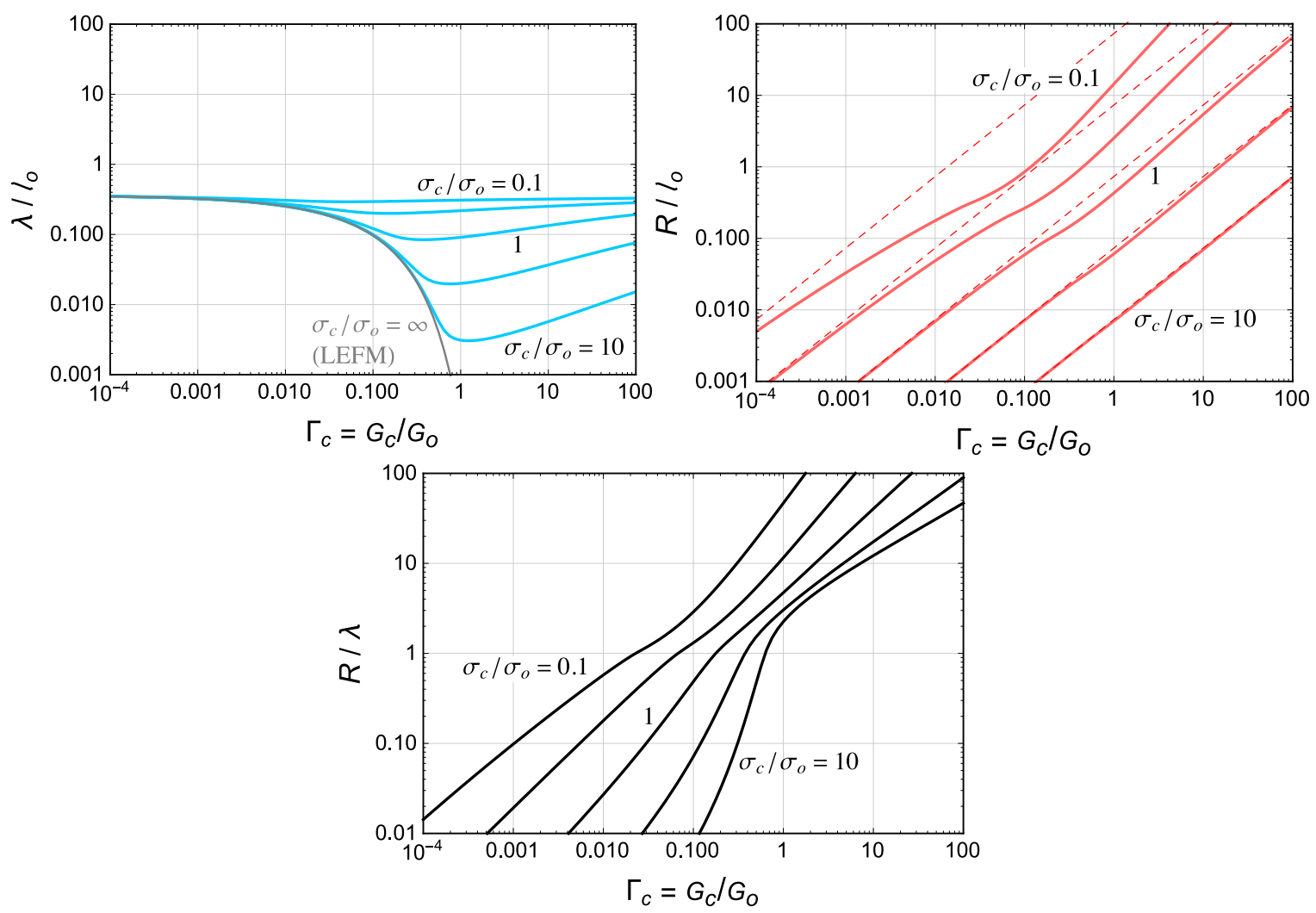

Figure 3: Extent of the fluid lag $\lambda$, cohesive zone length $R$, and the their ratio as a function of the normalized cohesive fracture energy $\Gamma_{c}$ at various values of the cohesive-to-confining stress ratio $\sigma_{c} / \sigma_{o}$ in $10^{0.5}$ increments on logarithmic scale. The LEFM solution $\left(\sigma_{c} / \sigma_{o}=\infty, R=0\right)$ for the lag and the dry-cohesive-zone-length $\left(R \approx 0.366 \ell_{c}[18]\right)$ solutions are shown by grey and red-dashed lines on respective plots.

\section{General solution for cohesive HF tip}

The HF tip solution which includes the coupling of the solid and fluid process zones can be thought of as the transition from the near-field given by either the cohesive $c$ or the lag $o$ asymptotes (as determined by the fracture energy partition) to the far-field zero-lag, zero-toughness $m$ asymptote. The other limiting asymptotic behaviors, i.e. $o$ or $c$ (depending on the near field type), and $k$, may or may not be realized in the solution depending on the separation of the relevant transitional scales.

For small $G_{c} / G_{o}$ we anticipate that the LEFM-based solution structure $k \underset{\ell_{k o}}{\rightarrow} \overrightarrow{\ell_{o}} m,(11)$, is generalized to

$$
G_{c} / G_{o} \ll 1: \quad c \underset{\ell_{c}}{\rightarrow} k \underset{\ell_{k o}}{\rightarrow} O \underset{\ell_{o}}{\rightarrow} m
$$

We note that (12) requires separation of the corresponding lengthscales, and, thus, the emergence of the intermediate $k$-asymptote $\left(\ell_{c} \ll \ell_{k o}\right)$ requires additional condition for a large enough cohesive stress $\sigma_{c} / \sigma_{o} \gg$ $\left(G_{c} / G_{o}\right)^{1 / 4}$. If the latter is not satisfied, then the boundary layer structure reduces to $c \rightarrow o \rightarrow m$.

For large $G_{c} / G_{o}$ the LEFM-based solution structure $k \underset{\ell_{k m}}{\rightarrow} m$ can be generalized in the presence of the cohesive zone to

$$
G_{c} / G_{O} \gg 1: \quad o \underset{\ell_{o}}{\rightarrow} m \underset{\ell_{m c}}{\rightarrow} \underset{\ell_{c}}{\rightarrow} k \underset{\ell_{k m}}{\rightarrow} m
$$

Again, we observe that the additional conditions for the emergence of the intermediate $m$ - and $k$ - asymptotes (i.e. $\ell_{o} \ll \ell_{m c}$ and $\left.\ell_{c} \ll \ell_{k m}\right)$ are $\left(G_{c} / G_{o}\right)^{1 / 4} \gg \sigma_{c} / \sigma_{o}$ and $\sigma_{c} / \sigma_{o} \gg\left(G_{c} / G_{o}\right)^{-1}$, respectively. If one of these conditions is not satisfied, the solutions structure reduces to $o \rightarrow c \rightarrow k \rightarrow m$ and $o \rightarrow m \rightarrow c \rightarrow m$, 

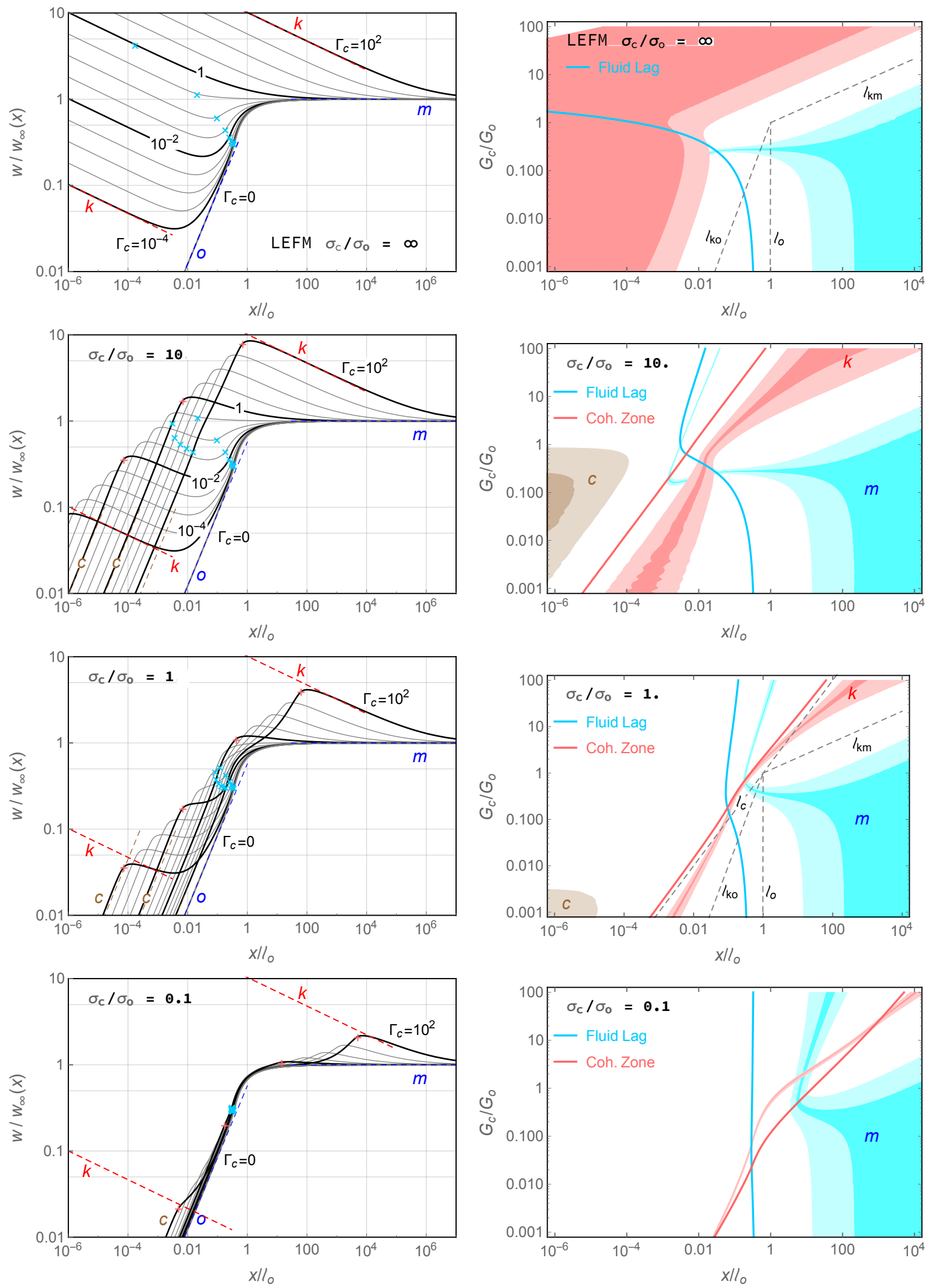

Figure 4: The crack opening normalized by the far field $m$-asymptote (left) and map of asymptotic regimes (right) for solutions shown in Figure 2. 
respectively. If both of the stated conditions are not satisfied, then the structure is expected to be given by $o \rightarrow c \rightarrow m$.

We obtain the numerical solution for the cohesive HF tip using an extension of the method of [18] based on Gauss-Chebyshev calculus. As further detailed in Appendix B, the extended method allows to solve the problem over extremely wide range of distances from the crack tip, which proves generally necessary to resolve the multiscale structure of the considered solutions. The solution for the net pressure and the crack opening distributions is shown in Figure 2a-d for four values of the cohesive stress ratio $\sigma_{c} / \sigma_{o}=\infty$ (LEFM), 10, 1 , and 0.1 , and various values of the fracture energy ratio $G_{c} / G_{o}$, while corresponding solutions for the crack opening normalized by the far-field $m$-asymptote are shown in Figure 4(left). The dependence of the fluid lag and process zone sizes on the problem parameters is show on Figure 3. The maps of the different asymptotic regions as function of the fracture energy ratio for solutions in Figure 2a-d are shown on Figure 4(right). There, the lighter (darker) colored regions correspond to $5 \%(1 \%)$ or less departure from the corresponding asymptote.

The striking feature of the solution at small to intermediate cohesive stress ratio (Figure 2d,c and Figure $4 \mathrm{~d}, \mathrm{c})$ is the nearly complete departure from the corresponding LEFM solution. In the case of small cohesive stress ratio, the solution is approximately given by the zero-toughness solution regardless of the toughness (cohesive fracture energy) value, unless the latter is very large $\left(G_{c} / G_{o}=100\right.$ in Figure $2 \mathrm{~d}$ ). These solutions lack the intermediate LEFM $k$-asymptote. This is further evidenced in the dependence of the fluid lag on the problem parameters (Figure 3a), in which the lag extent is much larger than suggested by the LEFM $\mathrm{HF}$ solution (grey line in Figure 3a), and is approximately given by its maximum (zero-toughness) value $\lambda / \ell_{o} \approx 0.3574$ for small values of the cohesive stress ratio.

It is only for large cohesive stress ratio (Figure 4b) do we see the emergence of the intermediate $k$ asymptote at moderate fracture energy values. As such, we established significant parametric constraints on the validity of the LEFM-based approaches (which replaces the cohesive zone with the point singularity) in the hydraulic fracture modeling.

\section{Evaluating effect of tip processes on finite $\mathrm{HF}$ propagation}

\subsection{Approximate Equation-of-Motion for a hydraulic fracture}

Consider the tip solution for the opening $w(x ; V)$ given as a function of the tip coordinate $x=\ell-X$ (moving with the crack tip) parametrized by the instantaneous tip velocity $V=d \ell / d t$ embedded in the tip solution scaling and the non-dimensional governing parameter(s). Here $X$ is the fixed coordinate emanating from the parent (finite) fracture center/fluid source and $\ell$ is the radius for the penny-shape fracture (Figure 1a) or the half-length for the plane-strain (KGD) fracture. An approximate continuation of this tip solution onto a finite fracture can be accomplished by substitution for the tip coordinate $x \Rightarrow\left(\ell^{2}-X^{2}\right) / 2 \ell$,

$$
w_{\text {finite }}(X, t) \approx w\left(\frac{\ell^{2}-X^{2}}{2 \ell} ; \frac{d \ell}{d t}\right)
$$

It is easily seen that (i) $w_{\text {finite }}(X)$ is equivalent to $w(x)$ asymptotically near the fracture front, (ii) $w_{\text {finite }}(X)$ possesses appropriate symmetry about $X=0$ (for the plane strain fracture case), and (iii) $w_{\text {finite }}(X)$ is in fact the exact expression for the fracture opening in the toughness-dominated LEFM case when $p=0$ uniformly along the crack. Using this continuation, the equation-of-motion (EoM) of a finite fracture, i.e. the equation governing trajectory $\ell(t)$, can be obtained from the global fluid balance in the fracture, which in the case of negligible fluid exchange between the fracture and the rock simply matches the injected fluid volume $V_{\text {inj }}(t)$ to the volume of the wetted part of the crack

$$
V_{\mathrm{inj}}(t)=2 \int_{0}^{\ell_{\mathrm{f}}(t)}(\pi X)^{d-1} w_{\text {finite }}(X, t) d X
$$

where $d$ is the "dimensionality" of the fracture ( $d=1$ for the plane-strain and $d=2$ for the radial fractures), and $\ell_{\mathrm{f}}=\ell-\lambda$ is the half-length/radius of the fluid-invaded part of the fracture. Eq. (15) with (14) provides an implicit ordinary differential equation to be solved for $\ell(t)$. Once the fracture trajectory is found, the 
corresponding approximate evolution of the fluid pressure can be obtained from numerical integration of the appropriate elasticity boundary integral equation.

In the context of the tip solution in a given scaling characterized by the length $\ell_{*}$ and opening $w_{*}$ scales (e.g. the $\ell_{o}$-scaling), let us denote the normalized lag $\Lambda=\lambda / \ell_{*}$ and opening $\hat{\Omega}(\hat{\xi})=w\left(\ell_{*} \hat{\xi}\right) / w_{*}$, where the normalized distance from the crack tip $\hat{\xi}=x / \ell_{*}$ is approximated as per the above continuation $\hat{\xi}(X) \approx\left(\ell^{2}-X^{2}\right) / 2 \ell \ell_{*}$. Then the EoM (15) with (14) can be written as

$$
\frac{V_{\mathrm{inj}}(t)}{\pi^{d-1} w_{*}\left(2 \ell_{*}\right)^{d}}=\hat{\xi}_{0}^{d / 2} F\left(\hat{\xi}_{0}, \hat{\xi}_{\mathrm{f}}\right) \quad \text { with } \quad F\left(\hat{\xi}_{0}, \hat{\xi}_{\mathrm{f}}\right) \equiv \int_{\hat{\xi}_{f}}^{\hat{\xi}_{0}} \frac{\hat{\Omega}(s)}{\left(\hat{\xi}_{0}-s\right)^{1-d / 2}} d s
$$

where

$$
\hat{\xi}_{0}=\hat{\xi}(X=0)=\frac{\ell}{2 \ell_{*}}, \quad \hat{\xi}_{\mathrm{f}}=\hat{\xi}\left(X=\ell_{\mathrm{f}}\right)=\frac{\ell^{2}-(\ell-\lambda)^{2}}{2 \ell \ell_{*}}=\Lambda-\frac{\Lambda^{2}}{4 \hat{\xi}_{0}} \approx \Lambda
$$

are the values of $\xi$ at the crack center (fluid source) and at the fluid front, respectively. Function $F$ can be tabulated from the tip solution, which in our case is parametrized by two non-dimensional tip parameters - the stress-ratio $\sigma_{c} / \sigma_{o}$ and the fracture energy ratio $\Gamma_{c}=G_{c} / G_{o}$. Recall from the definitions that the tip lengthscale $\ell_{o} \propto d \ell / d t$, opening scale $w_{o}=\left(\sigma_{o} / E^{\prime}\right) \ell_{o} \propto d \ell / d t$, and the energy ratio $\Gamma_{c} \propto 1 /(d \ell / d t)$ are functions of the instantaneous fracture tip velocity, and, thus, EoM (16) is an implicit ordinary differential equation in $\ell(t)$.

\subsection{EoM solutions for some limiting propagation regimes of radial $\mathrm{HF}$}

\subsubsection{LEFM, zero-lag case (the viscosity- to toughness- dominated evolution)}

Let us consider first a limiting case of the LEFM radial hydraulic fracture $\left(\sigma_{c} / \sigma_{o}=\infty\right)$ with negligible fluid lag $\left(\Gamma_{c} \gg 1\right)$. The tip solution corresponds to the $k \underset{\ell_{k m}}{\rightarrow} m$ transition with the distance from the crack tip where the corresponding transition lengthscale $\ell_{k m} \propto 1 / V^{2}$ is expected to be increasing function of time as the fracture front velocity $V=d \ell / d t$ diminishes with propagation distance. For radial HF driven by constant rate of fluid injection, the fracture radius $\ell(t)$ grows slower than the tip transition lengthscale $\ell_{k m}(t) \propto(d \ell / d t)^{-2}$ resulting in the evolution of the fracture regime from the viscosity-dominated at small times (when $\ell_{k m} \ll \ell$ ) to the toughness-dominated at large times (when $\ell_{k m} \gg \ell$ ) [21]. The viscosity-dominated $M$-solution and the toughness-dominated $K$-solution are given by Savitski and Detournay [22]. Application of the equation of motion to these two limiting cases, when the tip solution is restricted to the $m$ - and $k$-asymptotes for the opening, given by $\hat{\Omega}(\hat{\xi})=\beta_{m} \hat{\xi}^{2 / 3}$ and $\hat{\Omega}(\hat{\xi})=\beta_{k} \hat{\xi}^{1 / 2}$ in the $\ell_{k m}$-scaling, respectively, we can simplify the EoM as

$$
\frac{V_{\mathrm{inj}}(t)}{\pi w_{k m}\left(2 \ell_{k m}\right)^{2}}= \begin{cases}\frac{3 \beta_{m}}{5}\left(\frac{\ell}{2 \ell_{k m}}\right)^{1+5 / 3} & (M \text {-solution }) \\ \frac{2 \beta_{k}}{3}\left(\frac{\ell}{2 \ell_{k m}}\right)^{1+3 / 2} & (K \text {-solution })\end{cases}
$$

where $\ell_{k m}=\ell_{k}^{3} / \ell_{m}^{2}=G_{c}^{3} /\left[E^{\prime} \mu^{\prime 2}(d \ell / d t)^{2}\right]$ and $w_{k m}=\ell_{k}^{2} / \ell_{m}=G_{c}^{2} /\left[E^{\prime} \mu^{\prime}(d \ell / d t)\right]$. Considering constant rate of fluid injection $V_{\mathrm{inj}}=Q_{o} t$, and integrating the above ODE we get

$$
\ell_{M}(t) \approx \underbrace{\left(\frac{5}{2^{1 / 3} 3^{7 / 6} \pi}\right)^{1 / 3}}_{\gamma_{M} \approx 0.705} \times \underbrace{\left(\frac{E^{\prime} Q_{o}^{3}}{\mu^{\prime}}\right)^{1 / 6} t^{4 / 9}}_{L_{M}(t)=\text { dynamic lengthscale }}, \quad \ell_{K}(t) \approx \underbrace{\left(\frac{3}{2^{1 / 2} \pi}\right)^{2 / 5}}_{\gamma_{K} \approx 0.855} \times \underbrace{\left(\frac{E^{\prime 2} Q_{o}^{2}}{K^{\prime 2}}\right)^{1 / 6} t^{2 / 5}}_{L_{K}(t)=\text { dynamic lengthscale }}
$$

where we made use of the toughness parameter $K^{\prime}=\sqrt{32 / \pi} K_{I c}=\sqrt{(32 / \pi) G_{c} E^{\prime}}$ in order to be consistent with the prevalent notation in the literature. We note that the EoM $K$-solution is exact, while the EoM $M$-solution is within $1.5 \%$ of an accurate numerical solution $\gamma_{M \text {,num }} \approx 0.6955$ [22]. The latter providing a benchmark of the error associated with the EoM approximation.

As noted previously, the general solution in the considered case evolves from the $M$ to $K$ asymptotic solution with time [23]. The characteristic time $t_{M K}$ and length $L_{M K}$ scales of this evolution follow from contrasting the two asymptotes, $L_{M K}=L_{M}\left(t_{M K}\right)=L_{K}\left(t_{M K}\right)$, e.g. [12],

$$
t_{M K}=\left(\frac{\mu^{\prime 5} E^{\prime 13} Q_{o}^{3}}{K^{\prime 18}}\right)^{1 / 2} \quad L_{M K}=\frac{\mu^{\prime} E^{\prime 3} Q_{o}}{K^{\prime 4}}
$$


Using these radial HF transition scales, to introduce normalized radius $\gamma=\ell / L_{M K}$ and time $\tau=t / t_{M K}$, the EoM can be reduced to

$$
\tau=2 \pi\left(\gamma / \dot{\gamma}^{3}\right) F\left(\gamma \dot{\gamma}^{2} / 2,0\right)
$$

where $\dot{\gamma}=d \gamma / d \tau$ is the normalized crack front velocity and $F(\hat{\xi}, 0)=\int_{0}^{\hat{\xi}} \hat{\Omega}(s) d s$ is the normalized tip volume $\left(\hat{\Omega}(\hat{\xi})\right.$ is the $k \rightarrow m$ tip solution for the opening in the $\ell_{k m}$-scaling). The solution to the normalized EoM (see details in Appendix C), which governs the evolution between the early-time $\gamma=\gamma_{M} \tau^{4 / 9}$ and large-time $\gamma=\gamma_{K} \tau^{2 / 5}$ asymptotes, is shown on Figure 5a and on Figure 5b in the form normalized by the early-time $M$ -

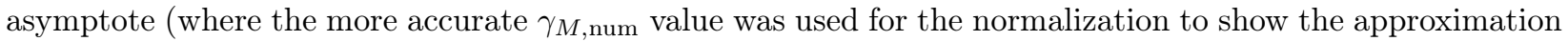
error of the EoM solution at early time). The symbols show an accurate numerical solution to the complete radial $\mathrm{HF}$ formulation [19].
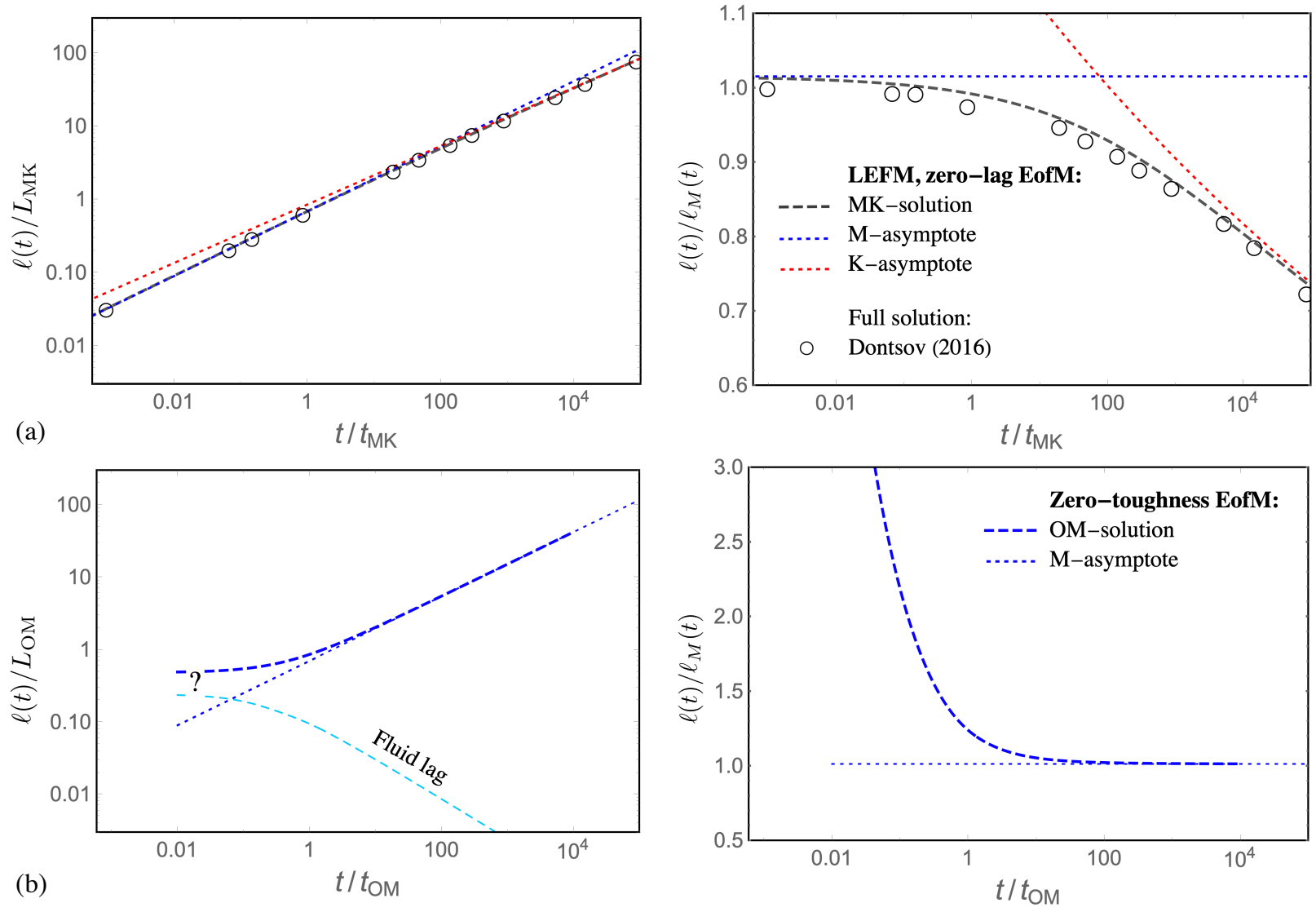

Figure 5: The MK, (a), and OM, (b), EoM solutions for the fracture radius in the corresponding transition scalings (left) and normalized by the $M$ asymptotic solution $\ell_{M} \propto t^{4 / 9}$ (right).

\subsubsection{Zero-toughness, non-zero lag case (the viscosity-dominated evolution of radial HF with the fluid lag)}

Another limiting case of the radial HF propagation of interest corresponds to the viscosity-dominated (zero toughness) fracture transitioning from the early-time solution influenced/dominated by the fluid lag [24, 25, $26]$ to the large-time zero-lag $M$-asymptotic solution. The corresponding tip solution is the $o \rightarrow m$ transition with the distance from the crack tip where the corresponding transition lengthscale $\ell_{o} \propto d \ell / d t$ is expected to be decreasing function of time as the fracture front velocity $V=d \ell / d t$ diminishes with propagation distance, leading to the aforementioned evolution of the fracture regime towards the zero-lag limit given by the $M$-solution. 
The characteristic time $t_{O M}$ of the transition towards the $M$ large-time asymptote is obtained by requiring that the lag $\lambda \sim \ell_{o}(t)$ is comparable to the crack length $\ell(t)$, i.e. $\ell_{o}\left(t_{O M}\right) \sim \ell\left(t_{O M}\right)$, while the corresponding transition lengthscale follows from using the $M$-solution, $L_{O M}=L_{M}\left(t_{O M}\right)$,

$$
t_{O M}=\frac{\mu^{\prime} E^{\prime 2} Q_{o}^{3}}{\sigma_{o}^{3}} \quad L_{O M}=\left(\frac{\mu^{\prime} E^{\prime 3} Q_{o}}{\sigma_{o}^{4}}\right)^{1 / 3}
$$

Using these radial HF transition scales, to introduce normalized radius $\gamma=\ell / L_{O M}$ and time $\tau=t / t_{O M}$, the EoM can be reduced to

$$
\tau=2 \pi \gamma \dot{\gamma}^{2} F\left(\frac{\gamma}{2 \dot{\gamma}}, \Lambda\right)
$$

where $F(\hat{\xi}, \Lambda)=\int_{\Lambda}^{\hat{\xi}} \hat{\Omega}(s) d s$ is the normalized tip volume $(\hat{\Omega}(\hat{\xi})$ is the $o \rightarrow m$ tip solution for the opening in the $\ell_{0}$-scaling and $\Lambda \approx 0.3574$ the corresponding value of the normalized lag). The solution to the EoM (see details in Appendix C) is seen to evolve towards the (zero-lag) $M$-solution at large time, while is fully affected by a fluid lag at early and intermediate times $t / t_{O M}$. At the early time, the EoM-predicted fluid lag values are a finite fraction $(\sim 0.25)$ the fracture radius (Figure $5 \mathrm{c}$ ), invalidating the underlining tip solution and putting in question the EoM solution in this time limit. (In fact, from rigorous consideration of the early time solution for a zero-toughness finite $\mathrm{HF}[24,26]$, it follows that the early time limit corresponds to a fast accelerating fracture almost devoid of fluid, i.e. $\lambda \approx \ell$ ).

\subsection{EoM solution for the propagation of a cohesive radial HF}

Finally, we use EoM to evaluate the non-LEFM, cohesive fracture effects on the propagation of a radial HF with a fluid lag. The EoM in the $O M$-scaling, $\gamma=\ell / L_{O M}$ and time $\tau=t / t_{O M}$, has the same form as that in the zero-toughness case, (19), where now, however, the normalized tip volume function $F$ and the normalized lag $\Lambda$ are dependent on the two tip non-dimensional parameters: the constant cohesive-to-stress ratio $\sigma_{c} / \sigma_{o}$ and velocity-dependent non-dimensional cohesive energy $\Gamma_{c}=G_{c} / G_{o}=\left(\sigma_{o} / E^{\prime}\right)\left(K_{I c}^{2} / E^{\prime}\right) /\left(\mu^{\prime}(d \ell / d t)\right)$. Using the $O M$-scaling, the latter can be written as

$$
\Gamma_{c}=\frac{\pi}{32} \frac{\mathscr{K}_{o m}}{\dot{\gamma}} \quad \text { where } \quad \mathscr{K}_{o m}=\frac{K^{\prime}}{E^{\prime 1 / 2} \sigma_{o}^{1 / 3} \mu^{\prime 1 / 6} Q_{o}^{1 / 6}}
$$

The toughness number $\mathscr{K}_{o m}$ can also be interpreted as a measure of the relative difference between the zerotoughness $(O M)$ and zero-lag $(M K)$ characteristic evolution scales for the radial HF: $\mathscr{K}_{o m}=\left(L_{O M} / L_{M K}\right)^{1 / 4}=$ $\left(t_{O M} / t_{M K}\right)^{1 / 9}$. Thus, the normalized solution of the EoM $\gamma=\gamma(\tau)$ is parametrized by two numbers $\mathscr{K}_{o m}$ and $\sigma_{c} / \sigma_{o}$.

We consider first the case of a high cohesive stress ratio, $\sigma_{c} / \sigma_{o}=10$, when the non-LEFM effects are expected to be minimal based on the analysis of the tip solution (Section 4). Indeed, in this case, Figure 6a shows a quick collapse of the solution trajectories (for different values of the radial HF toughness number $\mathscr{K}_{\text {om }}$ ) with time onto the corresponding LEFM, zero-lag $M K$ solution (shown by gray dashed lines) as the fluid lag (not shown) vanishes exponentially fast. At early times, the trajectories for small $\mathscr{K}_{\text {om }}$ values follow the zero-toughness $O M$-solution (shown by blue dashed line). The $M$ - and $K$-asymptotes are shown by dotted lines for reference. In the case of a low (relevant to the field applications) value of the cohesive stress ratio, $\sigma_{c} / \sigma_{o}=0.1$, the non-LEFM effects on the radial HF propagation are significant for all but the smallest value of the toughness number $\mathscr{K}_{o m}$ (Figure 6b), and, furthermore, dominate the fracture propagation at moderate-to-large values of $\mathscr{K}_{o m}$ (e.g. $\mathscr{K}_{o m}=1,2,3$, and 5). The larger cohesive stress solutions (Figure 6a), closely approximated by the LEFM $M K$ solution, predict slightly larger fracture run-out distance for low $\mathscr{K}_{o m}$ and grossly smaller fracture run-out for moderate $\mathscr{K}_{\text {om }}$ when compared to the small cohesive stress solutions (Figure 6b).

\section{Discussion}

\subsection{Field vs. lab hydraulic fracturing}

To gage the implications for the real physical systems, we consider estimates for typical sets of values of hydraulic fracture parameters relevant to the field and lab HF. Based on cleavage tests of sedimentary rocks 

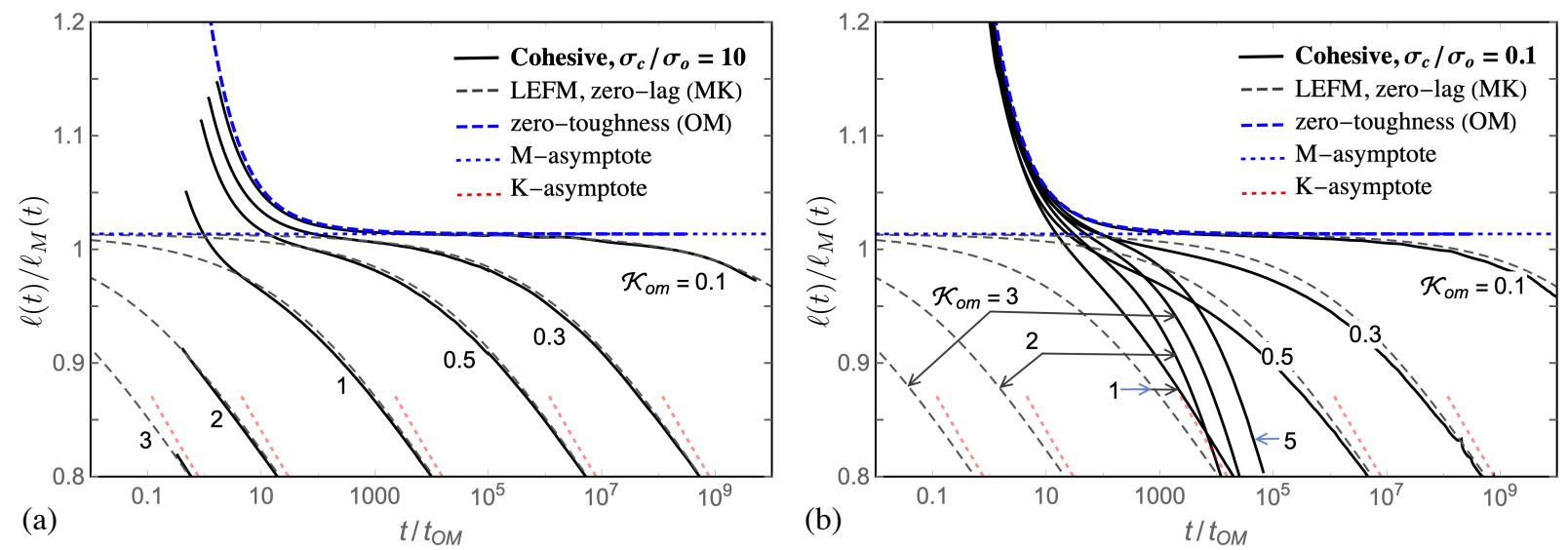

Figure 6: EoM solution for the evolution of the fracture radius normalized by the $M$-solution, $\ell(t) / \ell_{M}(t)$, for two values of the cohesive-to-confining stress ratio: (a) $\sigma_{c} / \sigma_{o}=10$ and low (b) $\sigma_{c} / \sigma_{o}=0.1$, and various values of the non-dimensional tougheness number $\mathscr{K}_{o m}$.

(limestones and sandstones) [27], we estimate for the peak cohesive stress $\sim 1$ to few $\mathrm{MPa}$ and for the cohesive fracture energy $G_{c} \sim 20$ to $300 \mathrm{~N} / \mathrm{m}$. Taking $\sigma_{c}=2 \mathrm{MPa}$ and $G_{c}=100 \mathrm{~N} / \mathrm{m}$ yields the critical cohesive crack opening $w_{c} \sim 100 \mu \mathrm{m}$ and cohesive lengthscale $\ell_{c} \sim 1.6 \mathrm{~m}$ (proxy for the extent of the cohesive zone in the fracture) when $E^{\prime}=33 \mathrm{GPa}$ is assumed. On the other hand, considering confining stress $\sigma_{o}=20 \mathrm{MPa}$, the fracture propagation velocity $V=0.1$ to $1 \mathrm{~m} / \mathrm{s}$ and fracturing fluid viscosity $\mu$ in the range between 5 cP ("slick water") and $100 \mathrm{cP}$ (conventional "gelled" fracturing fluid) yields for the lag opening scale $w_{o} \sim 0.5$ to $100 \mu \mathrm{m}$, the lag lengthscale $\ell_{o} \sim 0.001$ to $0.16 \mathrm{~m}$, and lag fracture energy $G_{o} \sim 10$ to $2000 \mathrm{~N} / \mathrm{m}$ (where lower/higher bounds correspond to lower/higher bounds of the product $\mu V$ ).

The above order of magnitude estimates suggest that small cohesive stress ratio $\sigma_{c} / \sigma_{o} \ll 1$ and small to moderate cohesive energy ratio $G_{c} / G_{o} \lesssim 10$ values for a field hydraulic fracture. Corresponding cohesive HF tip solution, see Figure 2d and corresponding asymptotic regimes map on Figure 4d, shows glaring absence of the LEFM $k$ asymptote from the solution.

Similar estimates and conclusions with regard to the prominence of the non-LEFM effects would apply for laboratory HF when a geologic material (rock) is fractured under the realistic (field) magnitude of the confining stress. On the other hand, a different outcome is expected for lab hydraulic fracture experiments in tough, brittle analog materials (PMMA, glass) characterized by order(s) of magnitude higher values of the peak cohesive strength $(\gtrsim 10 \mathrm{MPa})$ than rocks and by much smaller critical cohesive crack opening, which result in very small extent of the cohesive zone and predicted emergence of the intermediate LEFM $k$ asymptote at the fracture tip. The latter has indeed been observed in the laboratory HF experiments in analog solids [28].

\subsection{Limitations and proposed extensions to the current HF tip model}

We have shown that the coupling between the dissipation in the fluid flow, ultimately manifested in fracturing fluid lagging behind the fracture front, and the dissipation in breaking the rock over the cohesive process zone, is particularly prominent when the fluid enters the cohesive zone (i.e. fluid lag is embedded within the cohesive zone). Physically, the cohesive zone may represent the rough, partially-bridged part of the fracture, where the mean fracture opening $w$ is of the order of or smaller than the roughness of the fracture walls (scaled by the critical cohesive opening value $w_{c}$ ), thus, potentially questioning the validity of the classical Poiseuille flow law there [29]. Implementation of an amended, rough fracture flow near the HF tip is a part of ongoing work and may be expected to lead to enhanced fluid dissipation, therefore, modifying some of the findings reported here.

In the case of HF propagation in sufficiently permeable rock, the fluid exchange between the fracture and the rock would have to be accounted for. While the leak-off of the fracturing fluid into the surrounding rock can be effectively modeled within the framework of Carter's model in the context of zero fluid lag HF 
tip models (see for example [21]), the existence of the fluid lag, necessitated by fracture closure at the tip of a cohesive fracture as $w \propto x^{3 / 2}(x \rightarrow 0)$, would lead to suction (leak-in) of the formation pore fluid into the tip $[8,30]$, and modify the mechanical coupling there. Current model can be directly translated to the drained/slow regime of the HF tip propagation in permeable rock (when the fracturing fluid lag is filled by the infiltrated pore fluid at pressure equilibrated with the ambient formation pore pressure $p_{o}$ ) by replacing the net fluid pressure in the lag in the impermeable case, $p_{\text {lag }}=-\sigma_{o}$, by the drained permeable value, $p_{\text {lag }}=-\left(\sigma_{o}-p_{o}\right)$. Any departure from the drained HF tip propagation would have to explicitly consider the pore fluid exchange between the formation and the fracture [30,31, 32].

\section{Conclusions}

In this work we provide a systematic parametric study of the HF tip propagation problem accounting for the coupling of the solid and fluid process zones for the case of a fracture driven by a Newtonian fluid in an impermeable rock, while assuming the classical lubrication approximation for the fluid flow. We show through asymptotic analysis that the solution in general possesses an intricate structure supported by a number of nested lengthscales, on which different dissipation processes are realized. The latter processes can be cataloged as (1) dissipation in the fracture cohesive zone, ' $c$ ', parameterized by the fracture energy $G_{c}$ (cohesive energy release per unit fracture advance) and the peak cohesive stress $\sigma_{c}$ destroyed by fracturing; (2) the LEFM "reduction" of the cohesive zone process, ' $k$ ', quantified by $G_{c}$, but with the cohesive zone replaced by a singularity $\left(\sigma_{c} \rightarrow \infty\right)$; (3) viscous fluid dissipation associated with the fluid lag region, "o", parametrized by an equivalent fracture energy $G_{o}=\sigma_{o} w_{o}$, where $\sigma_{o}$ is the in situ confining stress (signifying the fracturing fluid pressure drop in the lag region from value $\sim \sigma_{o}$ to near zero) and $w_{o}$ is the corresponding fracture aperture scale; and (4) the viscous dissipation along the rest of the fracture (away from the fluid lag), ' $m$ '. The HF tip solution structure is bookended by the $c$ or $o$ (solid or fluid process zones) asymptotes near the tip and by the $m$ asymptote away from the tip, while the LEFM $k$ asymptote may emerge within the transitional region, as an intermediate asymptote, depending on values of the two governing parameters: the cohesive-to-lag fracture energy ratio $G_{c} / G_{o}$ and the cohesive-to-in-situ stress ratio $\sigma_{c} / \sigma_{o}$.

The full numerical solution of the HF tip problem in the parametric space shows that under field conditions prevailing in industrial applications of hydraulic fracturing, characterized by the in situ confining stress $\sigma_{o}$ in excess of the rock tensile strength $\sigma_{c}$ (peak cohesive stress), $\sigma_{o} \gtrsim \sigma_{c}$, the fluid lag is embedded in the fracture cohesive zone, and the LEFM intermediate ' $k$ ' asymptote does not exist. Thus, the explicit modeling of the cohesive zone in the hydraulic fracture is required in order to capture the solution.

Approximate solution for the propagation of an axisymmetric (penny-shape) HF from a point fluid source is constructed in order to demonstrate how the near cohesive HF tip effects (a semi-infinite HF model) translate to the propagation of finite hydraulic fractures. The solution method hinges on a suitable continuation of the tip solution onto the entire fracture, which leads to an ordinary differential 'equation-of-motion' for the fracture trajectory (fracture length as a function of time). The solution for unrealistically large (LEFMlike) cohesive-to-confining stress ratio $\left(\sigma_{c} / \sigma_{o}=10\right)$ is shown to overestimate the fracture run-out radius for the non-dimensional fracture toughness parameter $\mathscr{K}_{o m}=K^{\prime} / E^{\prime 1 / 2} \sigma_{o}^{1 / 3} \mu^{\prime 1 / 6} Q_{o}^{1 / 6}$ less than 1 and grossly underestimated the fracture radius for $\mathscr{K}_{o m} \gtrsim 1$.

\section{Acknowledgements}

The author acknowledges the support from the Ministry of Education and Science of the Russian Federation (Contract No. 14.581.21.0027, Unique identifier RFMEFI58117X0027). Thanks to Robert Viesca for interesting and valuable discussions related to this work.

\section{References}

[1] A. Peirce and E. Detournay. An implicit level set method for modeling hydraulically driven fractures. Computer Meth. Appl. Mech. Eng., 197:2858-2885, 2008. 
[2] A. Peirce. Modeling multi-scale processes in hydraulic fracture propagation using the implicit level set algorithm. Comput. Methods Appl. Mech. Engrg., 283:881-908, 2015.

[3] H. Zia and B. Lecampion. Explicit versus implicit front advancing schemes for the simulation of hydraulic fracture growth. Int. J. Num. Anal. Meth. Geomech., 43:1300-1315, 2018.

[4] G.I. Barenblatt. The mathematical theory of equilibrium cracks in brittle fracture. Adv. Appl. Mech., VII:55-129, 1962.

[5] D. S. Dugdale. Yielding of steel sheets containing slits. J. Mech. Phys. Solids, 8:100-104, 1960.

[6] Y. Ida. Cohesive force across the tip of a longitudinal-shear crack and Griffith's specific surface energy. J. Geophys. Res., 77:3796-3805, 1972.

[7] A. C. Palmer and J. R. Rice. The growth of slip surfaces in the progressive failure of over-consolidated clay. Proc. Roy. Soc. London, 433:469-477, 1973.

[8] A. M. Rubin. Tensile fracture of rock at high confining pressure: Implications for dike propagation. $J$. Geophys. Res., 98(B9):15,919-15,935, 1993.

[9] D. I. Garagash and E. Detournay. The tip region of a fluid-driven fracture in an elastic medium. ASME J. Appl. Mech., 67(1):183-192, 2000.

[10] J. Desroches, E. Detournay, B. Lenoach, P. Papanastasiou, J. R. A. Pearson, M. Thiercelin, and A. H-D. Cheng. The crack tip region in hydraulic fracturing. Proc. Roy. Soc. London, Serie A(447):39-48, 1994.

[11] J. R. Lister. Buoyancy-driven fluid fracture: The effects of material toughness and of low-viscosity precursors. J. Fluid Mech., 210:263-280, 1990.

[12] E. Detournay. Mechanics of hydraulic fractures. Annu. Rev. Fluid Mech., 48(311-339), 2016.

[13] J. R. Rice. Mathematical analysis in the mechanics of fracture. In H. Liebowitz, editor, Fracture, an Advanced Treatise, volume II, chapter 3, pages 191-311. Academic Press, New York NY, 1968.

[14] E. Sarris and P. Papanastasiou. The influence of the cohesive process zone in hydraulic fracturing modelling. Int. J. Fracture, 167:33-45, 2011.

[15] E. Sarris and P. Papanastasiou. Modeling of hydraulic fracturing in a poroelastic cohesive formation. Int. J. Geomechanics, 12:160-167, 2012.

[16] B. Carrier and S. Granet. Numerical modeling of hydraulic fracture problem in permeable medium using cohesive zone model. Engng. Fract. Mech., 79:312-328, 2012.

[17] Y. Yao, L. Liu, and L. M. Keer. Pore pressure cohesive zone modeling of hydraulic fracture in quasi-brittle rocks. Mechanics of Materials, 83:17-29, 2015.

[18] R. C. Viesca and D. I. Garagash. Numerical methods for coupled fracture problems. J. Mech. Phys. Solids, 113:13-34, 2018.

[19] E. V. Dontsov. An approximate solution for a penny-shaped hydraulic fracture that accounts for fracture toughness, fluid viscosity and leak-off. R. Soc. open sci., 3:160737, 2016.

[20] E. V. Dontsov. An approximate solution for a plane strain hydraulic fracture that accounts for fracture toughness, fluid viscosity, and leak-off. Int. J. Fracture, 205(2):221-237, 2017.

[21] D. I. Garagash, E. Detournay, and J. I. Adachi. Multiscale tip asymptotics in hydraulic fracture with leak-off. J. Fluid Mech., 669:260-297, 2011.

[22] A.A. Savitski and E. Detournay. Propagation of a fluid-driven penny-shaped fracture in an impermeable rock: Asymptotic solutions. Int. J. Solids Structures, 39(26):6311-6337, 2002. 
[23] M. Madyarova. Propagation of a penny-shaped hydraulic fracture in elastic rock. Master's thesis, University of Minnesota, Minneapolis, 2004.

[24] D. I. Garagash. Propagation of a plane-strain hydraulic fracture with a fluid lag: Early-time solution. Int. J. Solids Structures, 43:5811-5835, 2006.

[25] B. Lecampion and E. Detournay. An implicit algorithm for the propagation of a hydraulic fracture with a fluid lag. Comput. Methods Appl. Mech. Engrg., 196:4863-4880, 2007.

[26] A. P. Bunger and E. Detournay. Early-time solution for a radial hydraulic fracture. J. Eng. Mech., 133(5):534-540, 2007.

[27] R. G. Hoagland, G. T. Hahn, and A. R. Rosenfield. Influence of microstructure on fracture propagation in rock. Rock Mech., 5:77-106, 1973.

[28] A. P. Bunger and E. Detournay. Experimental validation of the tip asymptotics for a fluid-driven crack. J. Mech. Phys. Solids, 56:3101-3115, 2008.

[29] D. I. Garagash. Roughness-dominated hydraulic fracture propagation. EOS Trans. AGU (Fall Meeting Suppl.), 96(52):MR41A-2628, 2015.

[30] E. Detournay and D. Garagash. The tip region of a fluid-driven fracture in a permeable elastic solid. $J$. Fluid Mech., 494:1-32, 2003.

[31] Y. Kovalyshen. Fluid-Driven Fracture in Poroelastic Medium. PhD thesis, University of Minnesota, 2010.

[32] E. A. Kanin, D. I. Garagash, and A. A. Osiptsov. Near tip region of hydraulic fracture with pressuredependent leak-off. J. Fluid Mech., submitted, 2019.

\section{A Scaled equations}

\section{A.1 $\ell_{0}$-scaling}

Passing to non-dimensional field variables: distance from the tip $x / \ell_{o} \Rightarrow x$, lag $\lambda / \ell_{o} \Rightarrow \lambda$, net pressure $p / \sigma_{o} \Rightarrow p$, and crack opening $w / w_{o} \Rightarrow w$, where $\ell_{o}=\left(E^{\prime} / \sigma_{o}\right)^{3}\left(\mu^{\prime} V / E^{\prime}\right),(8)$, and $w_{o}=\left(\sigma_{o} / E^{\prime}\right) \ell_{o}=$ $\left(E^{\prime} / \sigma_{o}\right)^{2}\left(\mu^{\prime} V / E^{\prime}\right)$, leads to the scaled elasticity

$$
p(x)-\sigma_{\operatorname{coh}}(w(x))=\frac{1}{4 \pi} \int_{0}^{\infty} \frac{d w}{d s} \frac{d s}{x-s} \quad(x>0)
$$

and fluid flow

$$
p(x)=-1 \quad(0<x<\lambda), \quad 1=w^{2} \frac{d p}{d x} \quad(x>\lambda)
$$

equations. Scaled cohesive stress $\left(\sigma_{c o h} / \sigma_{o} \Rightarrow \sigma_{c o h}\right)$ can be expressed in terms of the scaled opening as

$$
\sigma_{c o h}\left(w<\Omega_{c}\right)=\Sigma_{c}\left(1-\frac{w}{\Omega_{c}}\right) \quad \sigma_{c o h}\left(w>\Omega_{c}\right)=0 \quad \text { with } \quad \Omega_{c}=\frac{2 \Gamma_{c}}{\Sigma_{c}}
$$

where, as previously defined in (10), $\Sigma_{c}=\sigma_{c} / \sigma_{o}$ and $\Gamma_{c}=\frac{1}{2}\left(\sigma_{c} w_{c}\right) /\left(\sigma_{o} w_{o}\right)$ are the non-dimensional peak cohesive stress and cohesive fracture energy, respectively, while $\Omega_{c}=w_{c} / w_{o}$ is the non-dimensional critical cohesive crack opening.

In the LEFM limit [9], the cohesive stress $\sigma_{c o h}(w)=\Gamma_{c} \delta_{\text {Dirac }}(w)$ drops out from the crack elasticity equation (20), while the normalized form of the LEFM crack tip propagation condition (3) becomes

$$
x \rightarrow 0: \quad w=\kappa \sqrt{x} \quad \text { with } \quad \kappa=\sqrt{32 \Gamma_{c} / \pi}
$$




\section{A.2 $\lambda$-scaling}

When solving the problem numerically, it is advantageous to rescale by the (unknown) lag, so that the scaled location for the fluid front is fixed $(x=1)$. In other words, replacing $\ell_{o}$ in the above scaling with $\lambda$,

$$
x / \lambda \Rightarrow x \quad \lambda / \ell_{o} \Rightarrow \lambda \quad p / \sigma_{o} \Rightarrow p \quad w /\left(\sigma_{o} / E^{\prime}\right) \lambda \Rightarrow w
$$

we have the scaled elasticity equation (20) unchanged and

$$
p(x)=-1 \quad(0<x<1), \quad \frac{1}{\lambda}=w^{2} \frac{d p}{d x} \quad(x>1)
$$

replacing (21). Scaled cohesive stress in (20) now takes form

$$
\sigma_{c o h}\left(w<\Omega_{c} / \lambda\right)=\Sigma_{c}\left(1-\frac{w}{\Omega_{c} / \lambda}\right) \quad \sigma_{c o h}\left(w>\Omega_{c} / \lambda\right)=0 \quad \text { with } \quad \Omega_{c}=\frac{2 \Gamma_{c}}{\Sigma_{c}}
$$

In the LEFM limit, the crack tip asymptote reads $w=\kappa \sqrt{x / \lambda}$ when $x \rightarrow 0$.

\section{B Gauss-Chebyshev method of solution}

\section{B.1 Non-singular crack with generalized mapping of semi-infinite crack domain into $(-1,1)$}

The method follows a familiar route [18], but with the generalized mapping of the semi-infinite crack $x \in$ $(0, \infty)$ into $z \in(-1,1)$

$$
x=x_{*}\left(\frac{1+z^{m_{*}}}{1-z^{m_{*}}}\right)^{m}
$$

where the map prefactor $x_{*}=\left(1-z_{i}^{m_{*}}\right)^{m} /\left(1+z_{i_{\lambda}}^{m_{*}}\right)^{m}$ is chosen such that the fluid front $x=1$ maps into the $i_{\lambda}$-node of the to-be-defined $z$-grid, i.e. $x\left[z_{i \lambda}\right]=1$. The original method [18] corresponds to the choice of exponents $m=m_{*}=1$. In the generalized map (26) and for the fixed $z$-grid, exponent $m$ larger or smaller than 1 allows to expand or shrink, respectively, the coverage of both the near $(x \ll 1)$ and far $(x \gg 1)$ fields of the semi-infinite domain. As seen in the following, a choice of odd natural number for $m$ (i.e. $m=1$, 3,5 , etc) allows to retain the use of GC-technique with $1 / 2$-fractional weight function. Similarly, exponent $m_{*}$ larger or smaller than 1 allows to refine (densify) or coarsen, respectively, the resulting $x(z)$-grid about $x=1$. This comes useful when solution has a weak discontinuity there, as is the case of the semi-infinite HF with the fluid lag, with the fluid front located at $x=1$ corresponding to the discontinuity of the pressure gradient. Map as defined by (26) requires the use of natural odd numbers for $m_{*} \geq 1$ (i.e. $m_{*}=1,3,5 \ldots$ ) or the reciprocals for $m_{*}<1$ (i.e. $m_{*}=1 / 3,1 / 5, \ldots$ ) in order to preserve the sign of $z$ in $z^{m_{*}}$. The latter condition can be relaxed by replacing $z^{m_{*}}$ with $\operatorname{sgn}(z)|z|^{m_{*}}$ in (26), although at the cost of reducing the differentiability of the map at $z=0$.

The mapped crack dislocation density can be written as

$$
\frac{d w}{d z}=\mathfrak{w}^{*}(z) F(z)
$$

where $\mathfrak{w}^{*}(z)$ embodies the near and far field asymptotic behavior of $d w / d z$ and $F(z)$ is a regular (bounded) function in $-1<z<1$. To make use of the GC-calculus, let us assume that $d w / d x \sim 1 / \sqrt{x}$ in the far and $\sim \sqrt{x}$ in the near field (which is the case of a non-singular crack), then an appropriate form of the function $\mathfrak{w}^{*}$ is

$$
\mathfrak{w}^{*}=z^{m_{*}-1} \frac{(1+z)^{3 m / 2-1}}{(1-z)^{m / 2+1}}
$$

Here the powers of $1 \pm z$ terms specify the requisite near and far field behavior. The prefactor $z^{m_{*}-1}$ has been introduced in order to regularize the elasticity integral (when $m_{*}>1$ ) near $z=s=0$ in the mapped domain. (Otherwise, $F(z)$ is necessarily zero at $z=0$, since the map derivative $x^{\prime}(z)$ vanishes there). 
The crack elasticity integral and propagation condition can be then written as

$$
\frac{1}{4 \pi} \int_{-1}^{1} \frac{\mathfrak{w}^{*}(s) F(s)}{x(z)-x(s)} d s=p(z)-\sigma_{c o h}(w(z))
$$

where we used a shorthand $p(x(z)) \Rightarrow p(z)$.

We use Gauss-Chebyshev discretization method with the weight-function $\mathfrak{w}(z)=\sqrt{(1+z) /(1-z)}$ to discretize $F_{j}=F\left(s_{j}\right), p_{i}=p\left(z_{i}\right)$, and $w_{i}=w\left(z_{i}\right)$ over the two sets of points $s_{j}=\cos (\pi(j-1 / 2) /(n+1 / 2))$, $j=1, \ldots, n$, and $z_{i}=\cos (\pi i /(n+1 / 2)), i=1, \ldots, n$, which correspond to the roots of the Chebyshev's third-kind $V_{n}(s)$ and fourth-kind $W_{n}(z)$ polynomials, respectively.

We can write the discretized set of governing equations in the $\lambda$-scaling as:

for the crack elasticity

$$
\frac{1}{4} \sum_{j=1}^{n} \mathcal{H}_{i j}^{*} F_{j}=p_{i}-\sigma_{c o h}\left(w_{i}\right)
$$
front)

in the fluid lag $\left(-1<z_{i} \leq z_{i_{\lambda}}\right.$ corresponding to $i \geq i_{\lambda}$ with $z_{i_{\lambda}}$ giving the mapped position of the fluid

$$
p_{i}=-1 \quad\left(i \geq i_{\lambda}\right)
$$

and in the fluid filled part of the crack $\left(z_{i_{\lambda}}<z_{i}<1\right.$ corresponding to $\left.i<i_{\lambda}\right)$

$$
\left(\frac{w^{2}}{d x / d z} \frac{d p}{d z}\right)_{i}=\frac{1}{\lambda}, \quad\left(\frac{d p}{d z}\right)_{i}=\sum_{i^{\prime}=1}^{n} \mathcal{D}_{i i^{\prime}} p_{i^{\prime}}^{\overleftarrow{ }} \quad\left(i<i_{\lambda}\right)
$$

In the above $p^{\leftarrow}(z)=p(z)+[d p / d z]_{z_{i_{\lambda}}-0}^{z_{\lambda}+0} H\left(z_{i_{\lambda}}-z\right)$ is "to-the-left" continuation of the net pressure function given in term of the pressure gradient jump at the fluid front in the mapped domain and Heaviside function [18]. $\mathcal{H}_{i j}$ and $\mathcal{D}_{i i^{\prime}}$ are the Hilbert transform and the $z$-grid differentiation matrices, respectively, given by

$$
\begin{gathered}
\mathcal{H}_{i j}^{*}=\frac{1}{\pi} \frac{\mathfrak{w}_{j}^{G C}}{x\left(z_{i}\right)-x\left(s_{j}\right)} \frac{\mathfrak{w}_{j}^{*}}{\mathfrak{w}_{j}} \\
\mathcal{D}_{i i^{\prime}\left(i \neq i^{\prime}\right)}=\frac{\omega_{i^{\prime}} / \omega_{i}}{z_{i}-z_{i^{\prime}}} \quad \mathcal{D}_{i i^{\prime}}=-\sum_{i^{\prime}=1, i^{\prime} \neq i}^{n} \mathcal{D}_{i i^{\prime}}
\end{gathered}
$$

where $\mathfrak{w}_{j}{ }^{G C}=\pi\left(1+s_{j}\right) /(n+1 / 2)$ are the G-C quadrature weights, and $\omega_{i}=(-1)^{i} \sin \left(\theta_{i} / 2\right) \sin \theta_{i}$ with $\theta_{i}=\arccos z_{i}$ are the weights of the barycentric form of Lagrange interpolation.

Finally, the crack opening on the $z$-grid $\left(w_{i}\right)$ is approximated by numerically integrating its gradient $(27)$ :

$$
w_{i}=\sum_{j=1}^{n} \mathcal{S}_{i j}^{*} F_{j}
$$

The integration matrix $\mathcal{S}_{i j}^{*}=\sum_{k=0}^{n-1} B_{k j} \Phi_{k}\left(z_{i}\right)$, with $B_{k j}=2 /(n+1 / 2) \cos \left(\theta_{j} / 2\right) \cos \left((k+1 / 2) \theta_{j}\right)\left(\theta_{j}=\right.$ $\left.\arccos s_{j}\right)$ and $\Phi_{k}(z)=\int_{-1}^{z} \mathfrak{w}^{*}(s) V_{k}(s)$, is obtained following the procedure of Section 2.4 of [18] while replacing $\mathfrak{w}(z) /(1-z)$ in [18]) by $\mathfrak{w}^{*}(z)$. ( $\Phi_{k}\left(z_{i}\right)$ are evaluated by numerical integration).

The system of $2 n$ equations (28)-(30) is complemented by the condition of the vanishing net fluid pressure at the far field

$$
p_{\mid x \rightarrow \infty} \approx \sum \mathcal{Q}_{i} p_{i}^{\leftarrow}=0
$$

where $\mathcal{Q}_{i}=-(-1)^{i} /(n+1 / 2) \cos \left(\theta_{i} / 2\right) \cot \left(\theta_{i} / 2\right)$ are the weights of the $z$-grid extrapolation to $z=1$ (following Section 2.2 of [18]). This yields an algebraic system of $2 n+1$ equations on $2 n+1$ unknowns: $F_{j}, p_{i}$, and $\lambda$.

Given that this defines a generalization of [18] numerical treatment of a semi-infinite crack, we point to the main differences from the original treatment: $\mathfrak{w}^{*}(z), \mathcal{H}_{i j}^{*}$, and $\mathcal{S}_{i j}^{*}$ replace $\mathfrak{w}(z) /(1-z)(w(z) /(1-z)$ in Viesca and garagash's notation), $\mathcal{H}_{i j}$ and $\mathcal{S}_{i j}$, respectively. 


\section{B.2 Singular crack with generalized mapping (26)}

In the case of a singular crack, the dislocation density has the LEFM-singularity at the crack tip, i.e. $d w / d x \sim$ $1 / \sqrt{x}$ when $x \rightarrow 0$. This prompts the following expression for the weight $\mathfrak{w}^{*}(z)$ in the decomposition $(27)$ for $d w / d z$ in the mapped domain

$$
\mathfrak{w}^{*}=z^{m_{*}-1} \frac{(1+z)^{m / 2-1}}{(1-z)^{m / 2+1}}
$$

and the use of the Gauss-Chebyshev discretization method with the weight-function $\mathfrak{w}(z)=1 / \sqrt{1-z^{2}}$ and corresponding set of points $s_{j}=\cos (\pi(j-1 / 2) / n), j=1, \ldots, n$, and $z_{i}=\cos (\pi i / n), i=1, \ldots, n-1$, which now correspond to the roots of the Chebyshev's first-kind $T_{n}(s)$ and second-kind $U_{n-1}(z)$ polynomials, respectively.

The rest of the numerical procedure laid out in Appendix B for the case of a non-singular crack applies here with the following amendments

- The sums over indices $i$ and $i^{\prime}$ (over the $z$-nodes) are now carried to $n-1$

- G-C quadrature weights are $\mathfrak{w}_{\mathfrak{j}}{ }^{G C}=n \pi$

- The weights of the barycentric form of Lagrange interpolation are $\omega_{i}=(-1)^{i} \sin ^{2}(\pi i / n)$

- Coefficients to calculate integration matrix $\mathcal{S}_{i j}^{*}=\sum_{k=0}^{n-1} B_{k j} \Phi_{k}\left(z_{i}\right)$ are given by $B_{k j}=\left(2^{H(k-1 / 2)} / n\right) \cos (k \pi(j-$ $1 / 2) / n)$ and $\Phi_{k}\left(z_{i}\right)=\int_{-1}^{z} \mathfrak{w}^{*}(s) T_{k}(s)$

- Extrapolation (to $z=1$ ) weights are $\mathcal{Q}_{i}=-(-1)^{i}\left(1+z_{i}\right)$

- The above procedure leads to $2(n-1)+1$ equation on $2 n$ unknowns $F_{j}$ (nunknowns), $p_{i}$ ( $n-1$ unknowns), and $\lambda$. The additional constrain is furnished by the LEFM-propagation condition, $d w / d x=\kappa /(2 \sqrt{\lambda x})$ when $x \rightarrow 0$, which translates to

$$
F(-1)=\kappa m m_{*}^{m / 2} \sqrt{x_{*} / \lambda}
$$

where $F(-1)$ can be found using the $s$-grid extrapolation to $s=-1: F(-1) \approx \sum_{j=1}^{n} \mathcal{P}_{j} F_{j}$ with weights $\mathcal{P}_{j}=\left((-1)^{j} / n\right) \tan (\pi(j-1 / 2) / n)$.

\section{B.3 Realization}

The realization of the above method with $m=3, m_{*}=2, i_{\lambda}=n / 2$ with $n=300$ nodes have been used to compute the numerical solutions reported in this work. The maps of the solution's asymptotic domains (Figures ...) validate the use of the cubic $(m=3)$ map for expanded spatial coverage. To appreciate this point, consider domain of the numerical solution $x \in\left[x\left(z_{n}\right), x\left(z_{1}\right)\right]$. For large $n$ and non-singular crack (i.e. $z$-grid corresponds to the roots of the Chebyshev 4th kind polynomial $W_{n}(z)$ as given in Appendix B.1), we have $z_{1} \approx 1-\pi^{2} / 2 n^{2}$ and $z_{n} \approx-1-\pi^{2} / 8 n^{2}$, and the solution domain is then approximately

$$
\left(\frac{\sqrt{m_{*}} \pi}{4} \frac{1}{n}\right)^{2 m} \leq \frac{x}{x_{*}} \leq\left(\frac{2}{\sqrt{m_{*}} \pi} n\right)^{2 m}
$$

It is apparent that the numerical domain bounds scale as $n^{ \pm 2 m}$, and thus depend very strongly on the map exponent $m$. For $n=300$ nodes, the numerical domain is approximately $x \in\left[2.6 \times 10^{-15}, 6.1 \times 10^{12}\right]$ for the cubic map $\left(m=3, m_{\lambda}=2\right)$ and $x \in\left[6.9 \times 10^{-6}, 3.6 \times 10^{4}\right]$ for the original "linear" map $\left(m=1, m_{\lambda}=1\right)$.

\section{Numerical method for solution of implicit EoM}

The fracture equation of motion for the normalized fracture length as a function of the normalized time in a given scaling, $\gamma=\gamma(\tau)$, has a general form (Section 5)

$$
\tau=\mathscr{F}(\gamma, d \gamma / d \tau)
$$


Given the implicit form of (31), the solution is obtained starting from some initial state $\gamma_{0}=\gamma\left(\tau_{0}\right)$ by, first, finding the given ( $i$ th) time step velocity as a root of (5), and, second, advancing the fracture front position

$$
\tau_{i}=\mathscr{F}\left(\gamma_{i},(d \gamma / d \tau)_{i}\right) \quad \Rightarrow \quad(d \gamma / d \tau)_{i} \quad \Rightarrow \quad \gamma_{i+1} / \gamma_{i}=\left(\tau_{i+1} / \tau_{i}\right)^{(d \ln \gamma / d \ln \tau)_{i}}
$$

Using a sufficiently refined logarithmic time stepping, this method allows to find the solution to a given degree of accuracy evaluated from the convergence of the solution with diminishing time step.

In the case of the limiting MK and OM solutions, the corresponding reduced EoMs (18) and (19), respectively, are separable, allowing to invert for the velocity, and then solve the resulting explicit ODE by standard methods. The separable/inverted forms of these EoMs are shown in Figure 7. The MK-EoM solution initiated at the early-time $M$-solution is straightforward. In the OM case, the EoM is non-unique and characterized by two branches with the "switch-back" at $\left(\tau / \gamma^{3}\right)_{*} \approx 1.5803$ and $(\dot{\gamma} / \gamma)_{*} \approx 0.2412$ (Figure 7(right)). The asymptotic analysis of the switch-back point allows to find the corresponding switch-back time and length, $\tau_{*} \approx 1.3817$ and $\gamma_{*} \approx 0.9562$. The EoM non-uniqueness is likely attributed to the breakdown of the EoM approximation when the corresponding fluid lag becomes a finite fraction of the fracture radius, which, at the switch-back, is $\lambda_{*} / L_{O M}=\Lambda \dot{\gamma}_{*} \approx 0.0824$, such that the lower/upper EoM branch in Figure 7 (right) corresponds to $\lambda$ less/greater than $\lambda_{*}$. The OM-solution is then obtained by integrating the two branches of the EoM forward and backward in time from the switch-back point. Figure 8 illustrates the such obtained OM-solution together with a number of other EoM solutions corresponding to perturbed initial condition applied at the switch-back point, i.e. when setting $\gamma\left(\tau_{*}\right) \gtrless \gamma_{*}$. The latter perturbed solutions are characterized by a kink in fracture radius and discontinuous value of the fluid lag at the switch-back.

In the general case of cohesive HF, the EoM is characterized by a similar switch-back behavior. However, the EoM is no-longer separable and can not be easily inverted. In this case, we use the method (32) to solve the EoM forward in time, while iterating on the initial condition until a converged solution is found numerically (equivalent to iterating on initial value $\gamma\left(\tau_{*}\right)$ in the illustration in Figure 8 for the OM-solution).
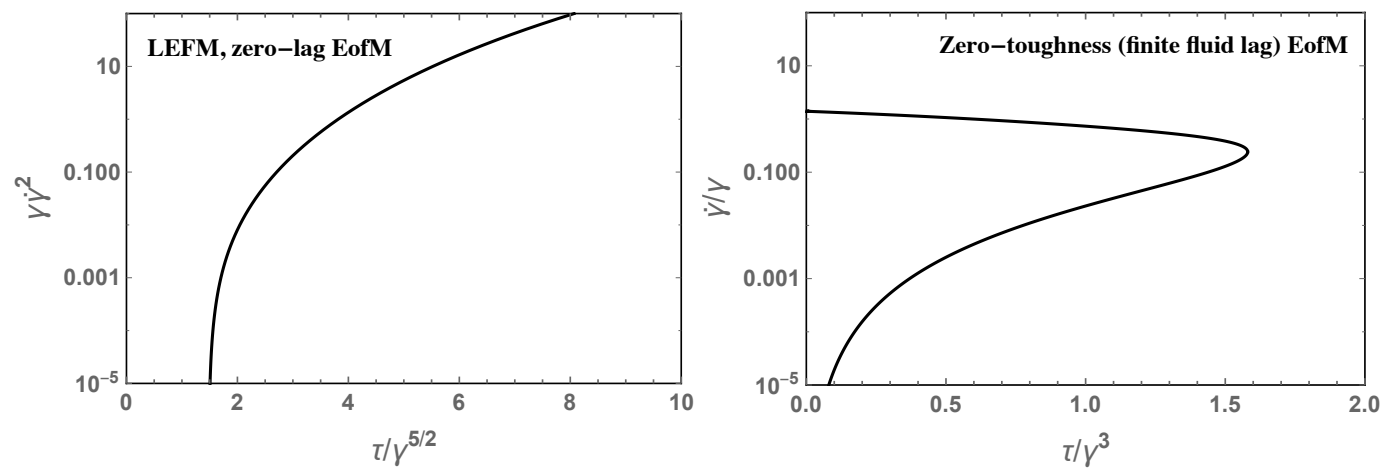

Figure 7: Explicit form of the Equation of Motion for (a) the LEFM, zero-lag (MK) and (b) the zerotoughness, finite lag $(\mathrm{OM})$ radial $\mathrm{HF}$. 

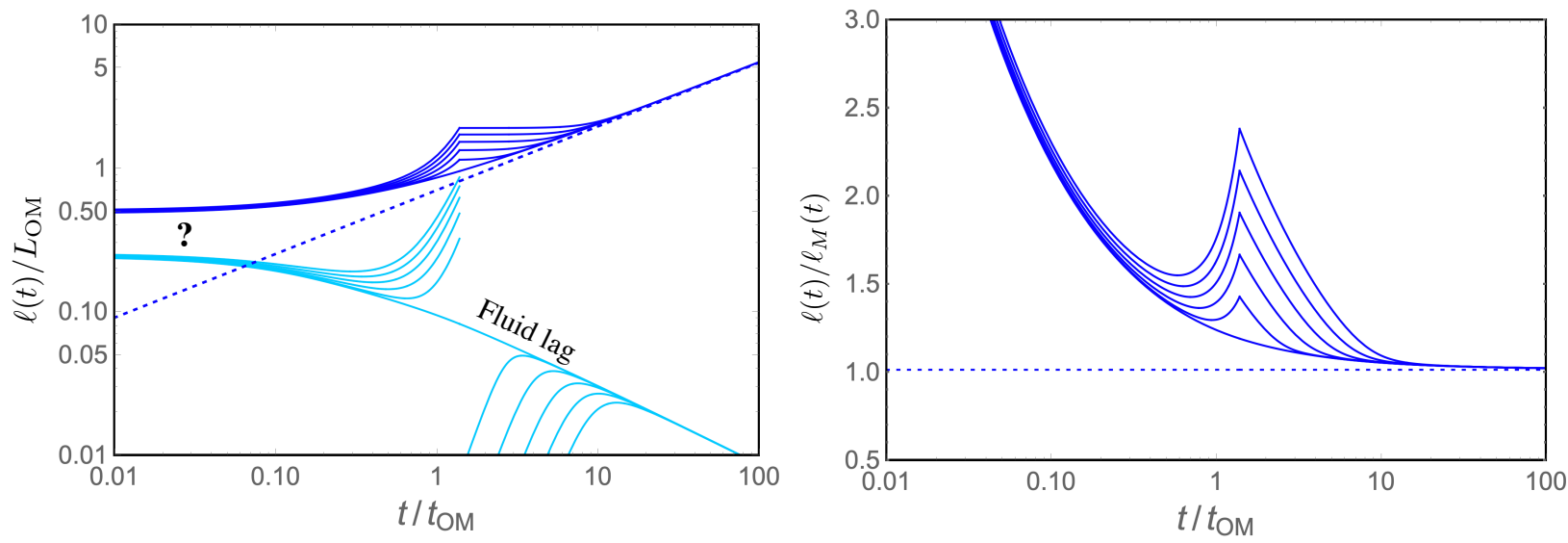

Figure 8: Sensitivity of the OM-solution to different "initial" condition at the EoM "switch-back time" $\left(t / t_{O M} \approx 1.3817\right)$ : fracture radius in the corresponding transition scaling, (left), and radius normalized by the $M$ asymptotic solution $\ell_{M} \propto t^{4 / 9}$, also shown by a dotted line, (right). 